\title{
Meta-analysis and systematic review of acupotomy combined with puncture and moxibustion in the treatment of knee osteoarthritis
}

\author{
Bing $\mathrm{Qu}^{1 \#}$, Xinyu $\mathrm{Wu}^{2 \#}$, Hongpeng $\mathrm{Liu}^{3}$, Weixin $\mathrm{Cai}^{4}$, Geqiang Wang ${ }^{4}$, Hanbing Song ${ }^{4}$, Fei Wang $^{5}$ \\ ${ }^{1}$ Division of CT and MRI, First Affiliated Hospital, Heilongjiang University of Chinese Medicine, Harbin, China; ${ }^{2}$ Basic Medical School, \\ Heilongjiang University of Traditional Chinese Medicine, Harbin, China; ${ }^{3}$ Third Orthopedics, Second Affiliated Hospital, Heilongjiang University \\ of Chinese Medicine, Harbin, China; ${ }^{4}$ Third Orthopedics, First Affiliated Hospital, Heilongjiang University of Chinese Medicine, Harbin, China; \\ ${ }^{5}$ Fourth Orthopedics, First Affiliated Hospital, Heilongjiang University of Chinese Medicine, Harbin, China \\ Contributions: (I) Conception and design: B Qu, X Wu, H Song, F Wang; (II) Administrative support: H Liu; (III) Provision of study materials or \\ patients: B Qu, X Wu, W Cai, G Wang, H Song; (IV) Collection and assembly of data: All authors; (V) Data analysis and interpretation: B Qu, H \\ Liu, F Wang; (VI) Manuscript writing: All authors; (VII) Final approval of manuscript: All authors. \\ "These authors contributed equally to this work. \\ Correspondence to: Hanbing Song; Fei Wang. First Affiliated Hospital, Heilongjiang University of Chinese Medicine, 26 Heping Rd, Harbin 150040, \\ China. Email: babutianlong125@163.com; wangfei631@126.com.
}

\begin{abstract}
Background: This study aimed to systematically evaluate the therapeutic effects of acupotomy combined with acupuncture and moxibustion on knee osteoarthritis (KOA), which was expected to provide a reference for clinical treatment of KOA using traditional Chinese medicine (TCM).

Methods: The databases PubMed, Embase, Medline, Ovid, and Springer were searched to retrieve randomized controlled trials (RCTs) on KOA treatment by acupotomy combined with acupuncture and moxibustion. The search time was set as from the date the database was established to 31 December 2020. The Cochrane Handbook for Systematic Reviews of Intervention 5.0.2 was used to conduct bias risk assessment on the included literature, and Review Manager 5.3 software was used for meta-analysis.

Results: A total of 10 RCTs were included in this study, including 1,073 participants. Meta-analysis results showed that compared with the control group, the clinical treatment efficiency of the experimental group was higher [mean difference $(\mathrm{MD})=5.72 ; 95 \%$ confidence interval $(\mathrm{CI}): 3.39$ to $9.64 ; \mathrm{Z}=6.54 ; \mathrm{P}<0.00001$ ], and the postoperative visual analogue scale (VAS) scores were reduced (MD $=-1.72 ; 95 \% \mathrm{CI}:-2.41$ to -1.03 ; $\mathrm{Z}=4.86 ; \mathrm{P}<0.00001)$.
\end{abstract}

Discussion: Acupotomy combined with acupuncture and moxibustion treatment for KOA can increase clinical treatment efficiency, and relieve postoperative pain, suggesting that the combination of acupotomy, acupuncture, and moxibustion has better therapeutic effects on KOA and can be promoted clinically.

Keywords: Acupotomy; acupuncture and moxibustion; knee osteoarthritis (KOA); meta-analysis

Submitted Apr 11, 2021. Accepted for publication Jun 11, 2021.

doi: 10.21037/apm-21-1083

View this article at: https://dx.doi.org/10.21037/apm-21-1083

\section{Introduction}

Knee osteoarthritis (KOA) is the most common area of osteoarthritis (OA), and is mainly characterized by the progressive degeneration and destruction of cartilage, articular cartilage repair, osteophyte formation, subchondral bone remodeling, and so on (1). The elderly are predominantly affected by KOA, and it is more common in women than men. Patients with KOA usually have symptoms such as knee pain, swelling, and joint snapping. If not treated in time, it will affect the knee joint function and the patient's quality of life (2). At present, the treatment methods for KOA include drug therapy, non-drug therapy, additional therapy, and surgical treatment. Non-surgical therapy is routinely applied. Drug therapy can be delivered 
via injection of anti-inflammatory analgesics or sodium hyaluronate into the joint cavity, but it is difficult to achieve the expected therapeutic effect (3).

Acupotomy and acupuncture and moxibustion are commonly used methods of traditional Chinese medicine (TCM) for the treatment of KOA. Acupuncture and moxibustion includes simple acupuncture, moxibustion, warm needle moxibustion, electric acupuncture, fire needle, and acupuncture point injection. Among them, moxibustion is an important TCM treatment. The moxa is prepared by drying the leaves and kneading to remove impurities, and then moxa is made into moxa sticks. The moxa is ignited to generate heat to stimulate specific acupoints, and to reinforce essence to adjust the function disorder. Clinical studies have shown that the combined application of acupotomy, acupuncture, and moxibustion can effectively relieve cartilage tissue pain, reduce pressure in the patient's knee joint, and restore the movement function of the knee joint (4). Though there is increasing research on the treatment of KOA with acupotomy and acupuncture and moxibustion, the systematic efficacy and quality evaluation of research is lacking. As a result, it is difficult to objectively reflect the therapeutic effects of acupotomy and acupuncture and moxibustion on KOA. Based on the reasons above, we adopted meta-analysis to systematically evaluate randomized controlled trials (RCTs) of acupotomy combined with acupuncture and moxibustion for the treatment of KOA.

We present the following article in accordance with the PRISMA reporting checklist (available at https://dx.doi. org/10.21037/apm-21-1083).

\section{Methods}

\section{Literature retrieve}

A total of 5 English databases (PubMed, Embase, Ovid, Medline, and Springer) were used to retrieve literature on the treatment of KOA by acupotomy combined with acupuncture and moxibustion. The retrieval time was set as from the establishment of each database to 31 December 2020. The search was performed using a combination of subject terms and free words, including "Knee Osteoarthritis", "KOA", "acupotomy", "acupuncture and moxibustion", "needle knife", and "acupotomology". The search terms were connected with "AND" or "OR". The retrieval was carried out by 2 researchers independently.

\section{The inclusion and exclusion criteria}

The inclusion criteria were as follows: (I) participants were patients who had been clinically diagnosed with KOA, unrestricted by gender and source; (II) RCT research published in English databases; (III) for literature of acupotomy treatment for KOA, the experimental group accepted acupotomy therapy, and the control group accepted other therapies. For literature of acupotomy combined with acupuncture and moxibustion for KOA, the experimental group accepted the combined treatment, and the control group accepted other treatment methods; (IV) There were clear inclusion criteria, diagnostic criteria, and therapeutic effects evaluation criteria for participants; (V) evaluation indexes included clinical treatment efficiency and visual analogue scale (VAS) score.

The exclusion criteria were as follows: (I) retrospective analyses, case reports, cohort studies, and other non-RCTs; (II) non-English literature or unpublished literature; (III) participants were ROA patients or patients with ROA combined with other diseases; (IV) literature with animals as research subjects; (V) literature published in duplicate; (VI) literature with incomplete research data so that it was impossible to calculate the corresponding index.

\section{Literature screening}

The software Endnote X6 (Clarivate Analytics, Philadelphia, $\mathrm{PA}$, USA) was used to build a database and remove repetitive literature. The remaining literature were then screened by 2 senior experts. Literature that was not related to this research was excluded first through reading the title and abstract. Literature that did not meet the requirements was excluded further by reading the full text. The quality evaluation was performed next. If there were inconsistencies between the 2 experts, a consensus conclusion was obtained either through discussion, or a third expert was invited to arbitrate.

\section{Data extraction}

The 2 experts used a unified Excel table to extract data independently. We then performed 3 preliminary experiments before data extraction. If there were inconsistencies among experts, a consensus conclusion was reached through discussion, or a third expert was invited to arbitrate. The data extracted included: (I) research title; (II) first author and year of publication; (III) name of the 
publication; (IV) The publication time of the research; (V) basic information of research participants: average age, gender, treatment plan, and drug dosage; (VI) grouping method and statistical method of the experimental group and the control group; (VII) source, sample size, and outcome indexes of the cases.

\section{Literature quality evaluation}

A risk assessment of bias was conducted at the same time by 2 researchers. If there were inconsistencies, the results were determined through discussion, or a third expert was invited for arbitration. The quality evaluation of this study referred to the standards in the Cochrane Handbook for Systematic Reviews of Intervention 5.0.2 (https:// training.cochrane.org/handbook/archive/v5.0.2/), and the evaluation content included whether the random method was described properly; whether allocation concealment was adopted; whether the blind method was used for subjects; whether the blind method was used for the outcome assessor; whether the research data was complete; whether there was a selective report; and whether there were other biases.

\section{Statistics}

The software STATA 11.0 (StataCorp LLC., College Station, TX, USA) was used to analyze the included literature, and Review Manager 5.3 (RevMan, Copenhagen, The Nordic Cochrane Center, The Cochrane Collaboration, 2014) was used for meta-analysis and to draw the forest plot. The publication bias of the literature was analyzed by funnel chart. For binary variables such as clinical treatment efficiency, the odds ratio (OR) was used as the effect size, with the $95 \%$ confidence interval $(95 \%$ CI) calculated. For continuous variables such as VAS score, Western Ontario and McMaster Universities Osteoarthritis Index (WOMAC) score, and interleukin-6 6 (IL-6 $\beta$ ) level, if the detection indexes had the same unit, the weighted mean difference (MD) was used as the effect size, and if the units were different, the standardized MD (SMD) was used as the effect size. The $\mathrm{I}^{2}$ test was used to evaluate the heterogeneity of the included literature. If $\mathrm{I}^{2}>50 \%$, it meant that there was large heterogeneity between the studies. The random effects model (REM) was used for meta-analysis. If $\mathrm{I}^{2}<50 \%$, it meant that the homogeneity between the studies was relatively good, and the fixed effects model (FEM) was used for meta-analysis of the combined effect size. The combined effect size test was performed using $\mathrm{U}$ test and $95 \% \mathrm{CI}$, and the result of $\mathrm{U}$ test was expressed by $\mathrm{P}$ value. A $\mathrm{P}$ value $<0.05$ was the threshold for significance.

\section{Results}

\section{Meta-analysis of acupotomy treatment for $\mathrm{KO} A$}

\section{Basic information of included literature}

A total of 936 related references were obtained in the preliminary retrieve, among which 383 were retrieved from PubMed, 231 from Embase, 166 from Ovid, 94 from Medline, and 62 from Springer. After the preliminary search, the titles of the references were input into the Endnote X6 software, and 188 duplicates were eliminated. We then invited 2 researchers to read the title and the abstract section of the literature. A total of 336 references remained according to the inclusion and exclusion criteria. Finally, 323 articles were further eliminated after having their full texts read carefully and cross-examined, with a total of 13 references (5-17) identified. The publication time was from 2007 to 2020, including 1,073 cases, with 536 in the experimental group and 537 in the control group. The baseline data such as age and disease course of the 2 groups were comparable (Figure 1, Table 1).

\section{The bias risk assessment of included literature}

The Cochrane tool was used to evaluate the bias risk of the 13 references included in this study, and then the bias risk map was drawn using Review Manager 5.3 software, as shown in Figures 2 and 3. The risk of bias assessment included 7 items: (I) whether a random sequence was used; (II) whether allocation concealment was used; (III) whether the blind method was used for the subjects; (IV) whether the blind method was used for the outcome assessor; (V) whether the research data was complete; (VI) whether it was a selective report; (VII) whether there were other biases.

\section{The effective rate of acupotomy for KOA}

A total of 11 references with a total of 970 KOA patients included analyzed the effective rate of clinical treatment of patients, of which 480 were in the experimental group, with 429 effective cases, and 490 were in the control group, with 402 effective cases. The heterogeneity test results $\left(\mathrm{I}^{2}=78 \%\right.$, $\mathrm{P}<0.00001)$ indicated that there was a certain degree of heterogeneity among the studies. Hence, the REM was used for analysis, and the analysis results are shown in 


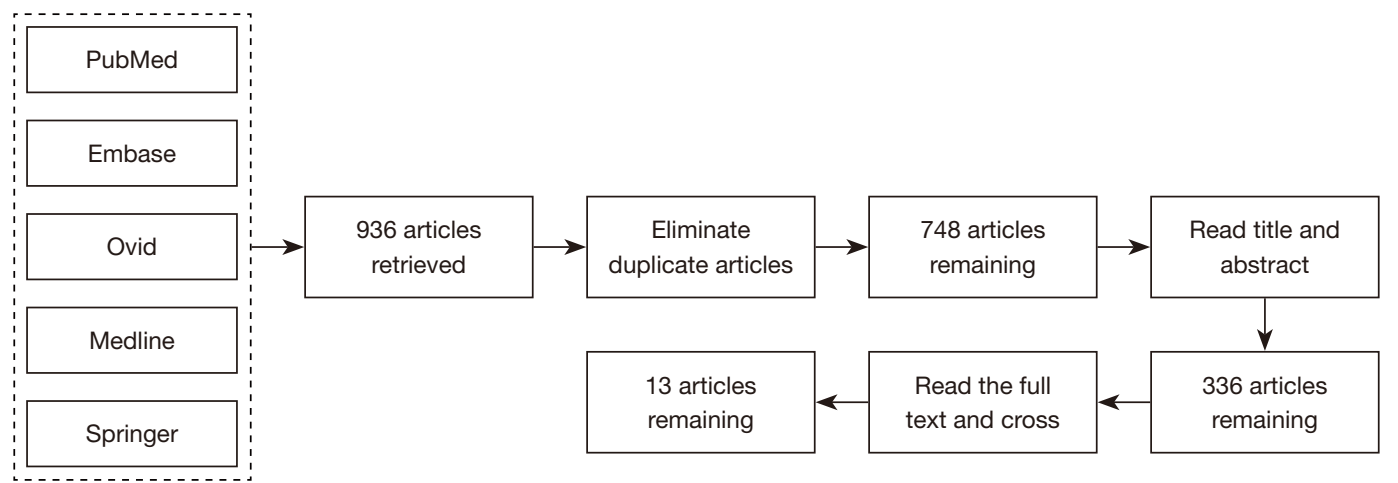

Figure 1 Literature retrieve process of acupotomy treatment for KOA. KOA, knee osteoarthritis.

Table 1 Basic characteristics of the included literature

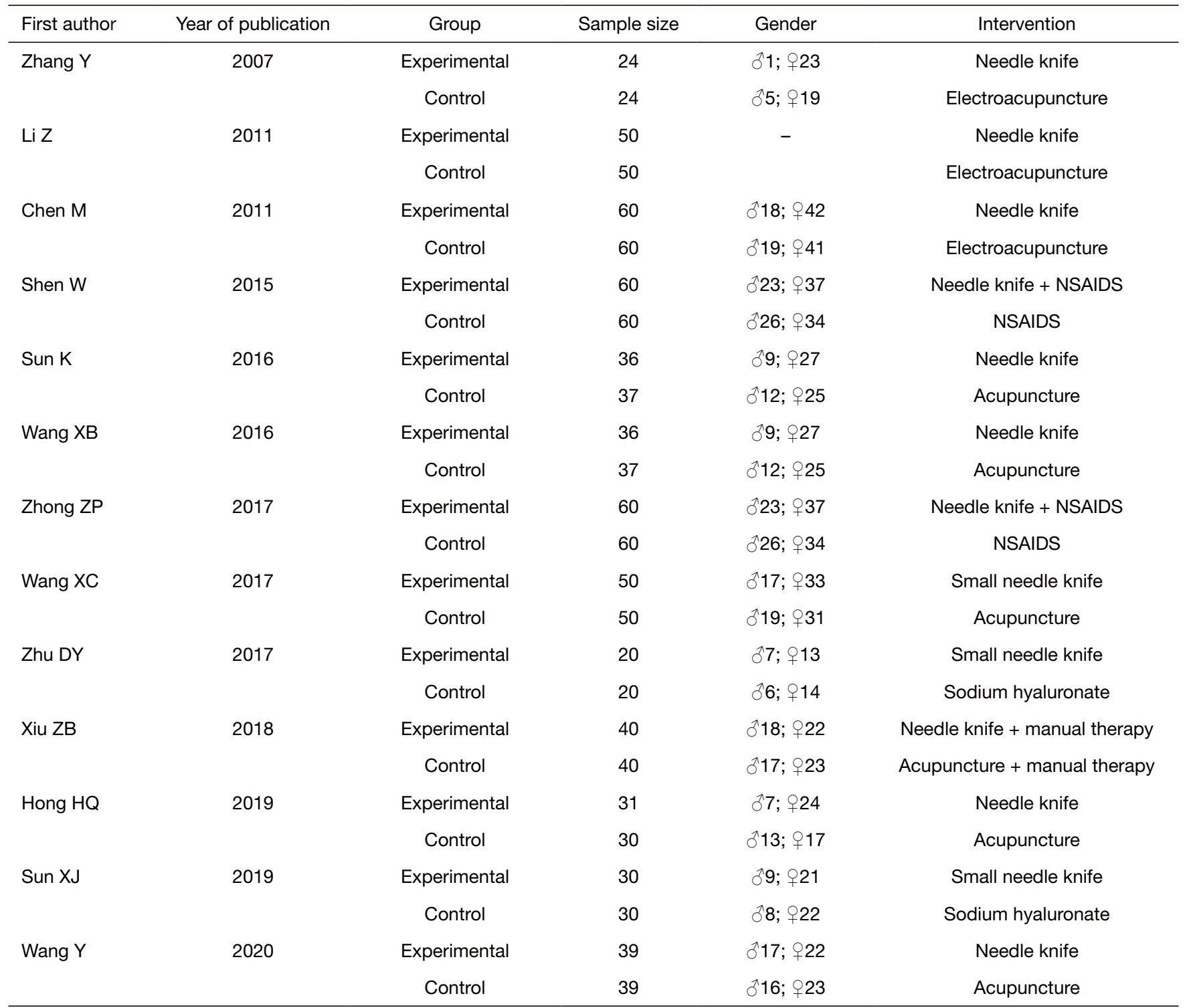

NSAIDS, non-steroidal anti-inflammatory drugs. 


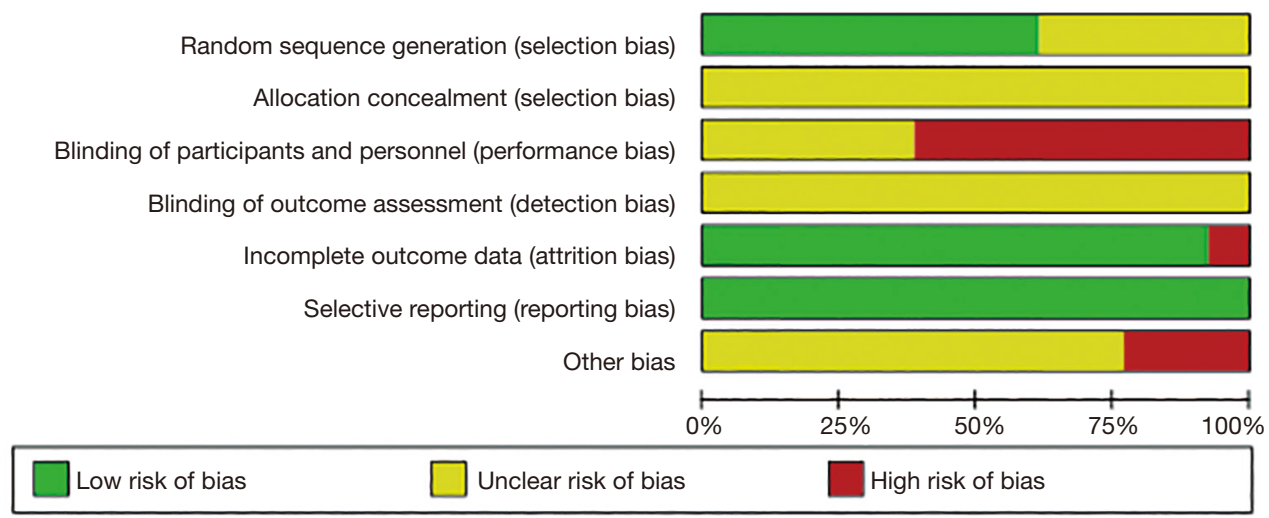

Figure 2 Meta-analysis bias risk assessment bar graph of acupotomy treatment of KOA. KOA, knee osteoarthritis.

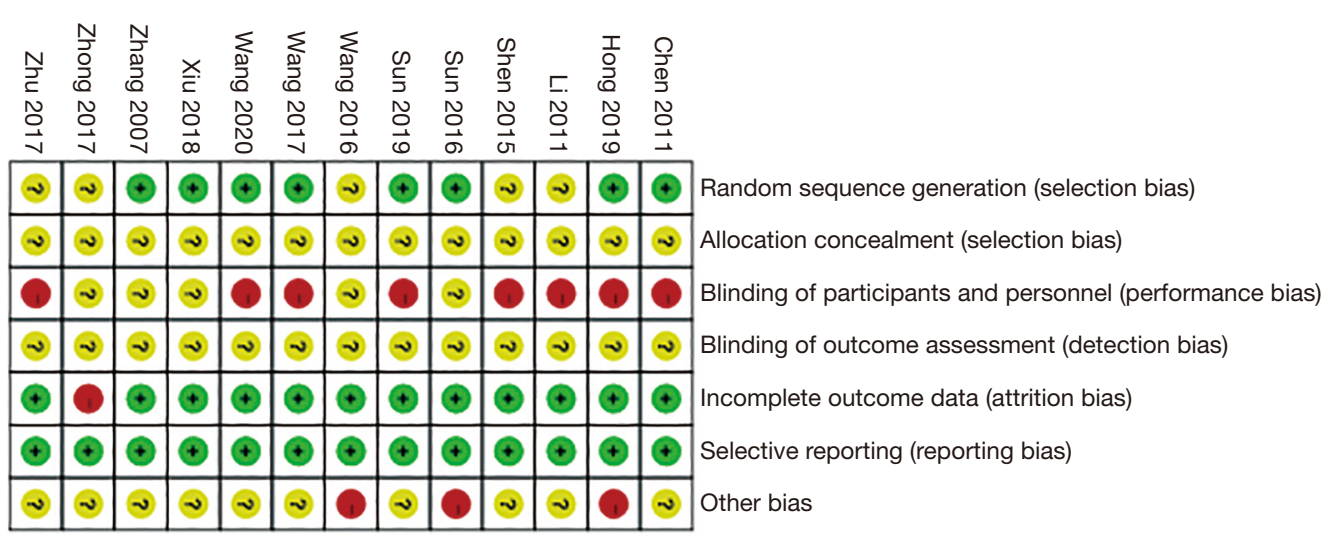

Figure 3 Meta-analysis bias risk assessment graph of acupotomy treatment of KOA. KOA, knee osteoarthritis.

\begin{tabular}{|c|c|c|c|c|c|c|c|c|c|c|}
\hline \multirow[b]{2}{*}{ Study or Subgroup } & \multicolumn{2}{|c|}{ Experimental } & \multicolumn{2}{|c|}{ Control } & \multicolumn{2}{|c|}{ Odds Ratio } & \multirow{2}{*}{\multicolumn{3}{|c|}{$\begin{array}{c}\text { Odds Ratio } \\
\mathrm{M}-\mathrm{H} . \text { Randem, } 25 \% \mathrm{Cl}\end{array}$}} & \\
\hline & Exents & Total & Exants & Total & Wcight & $\mathrm{M} \cdot \mathrm{H}$, Random, $95 \% \mathrm{Cl}$ & & & & \\
\hline Chen 2011 & 58 & 60 & 54 & 60 & $9.5 \%$ & $3.22(0.62,16.66)$ & & & & \\
\hline Horg 2019 & 29 & 31 & 22 & 30 & $9.5 \%$ & $5.27[1.02,27.33]$ & & & & \\
\hline Li 2011 & 47 & 50 & 41 & 60 & $10.5 \%$ & $7.26(2.00,26.31)$ & & & & \\
\hline Shen 2015 & 54 & 60 & 50 & 60 & $11.0 \%$ & $1.80[0.61,5.32]$ & & & & \\
\hline Sun 2016 & 34 & 36 & 34 & 37 & $8.9 \%$ & $1.50(0.24,9.55)$ & & & & \\
\hline Sun 2019 & 29 & 30 & 20 & 30 & $8.1 \%$ & $14.50[1.72,122.40]$ & & & & \\
\hline Wang 2017 & 50 & 50 & 45 & 50 & $6.2 \%$ & $12.21[0.66 .226 .97]$ & & & & \\
\hline Wang 2020 & 38 & 39 & 31 & 39 & $8.1 \%$ & $9.81[1.16,82.71]$ & & & & \\
\hline Xiu 2018 & 38 & 40 & 32 & 40 & $9.5 \%$ & $4.75[0.94,23.98]$ & & & & \\
\hline Zhang 2007 & 23 & 24 & 22 & 24 & $7.2 \%$ & $2.09[0.18,24.73]$ & & & & \\
\hline Zhong 2017 & 29 & 60 & 51 & 60 & $11.5 \%$ & $0.17[0.07,0.39]$ & & & & \\
\hline Total $(95 \% \mathrm{Cl})$ & & 480 & & 490 & $100.0 \%$ & $3.09[1.11,8.56]$ & & & & \\
\hline Total events & 429 & & 402 & & & & & & & \\
\hline $\begin{array}{l}\text { Heterogeneity: Tau' } \\
\text { Test for overall effect }\end{array}$ & $\begin{array}{l}\text { 2.15; Chi } \\
=2.17\langle P\end{array}$ & $\begin{array}{l}=44.73 \\
=0.03\rangle\end{array}$ & $d f=10\langle P$ & $P<0.00$ & $0001) ; 1$ & & 0.001 & $\begin{array}{c}0.1 \\
\text { perimental] }\end{array}$ & $\begin{array}{lr}1 & 10 \\
\text { [control] }\end{array}$ & $1000^{\circ}$ \\
\hline
\end{tabular}

Figure 4 Forest plot of clinical treatment effective rate of acupotomy for KOA. KOA, knee osteoarthritis; HR, hazard ratio; CI, confidence interval.

Figure 4. The combined effect size of the meta-analysis was $(\mathrm{MD}=3.09 ; 95 \% \mathrm{CI}: 1.11$ to $8.56 ; \mathrm{Z}=2.17 ; \mathrm{P}=0.03)$, and the diamond in the forest diagram was located on the right side of the vertical line, which suggested that the clinical effective rate of acupotomy treatment was higher than that of the control group. 


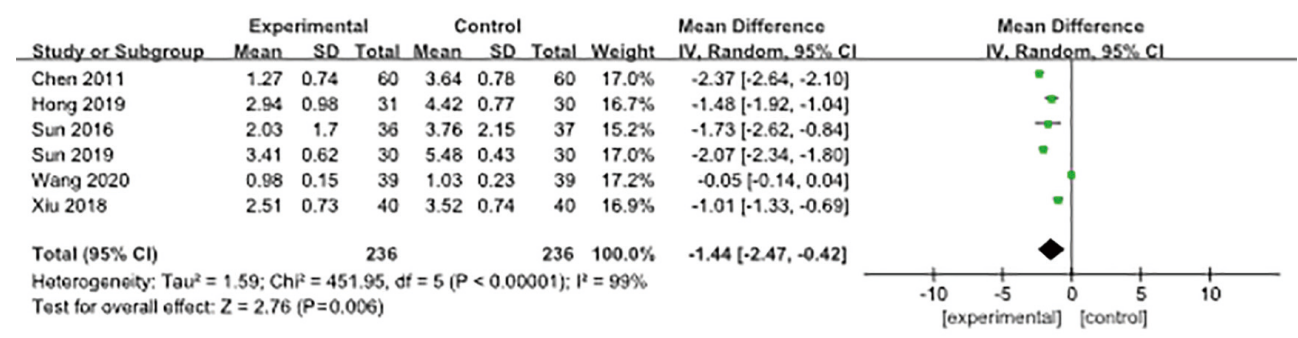

Figure 5 Forest plot of VAS score after acupotomy treatment of KOA. VAS, visual analogue scale; KOA, knee osteoarthritis; CI, confidence interval; SD, standard deviation.

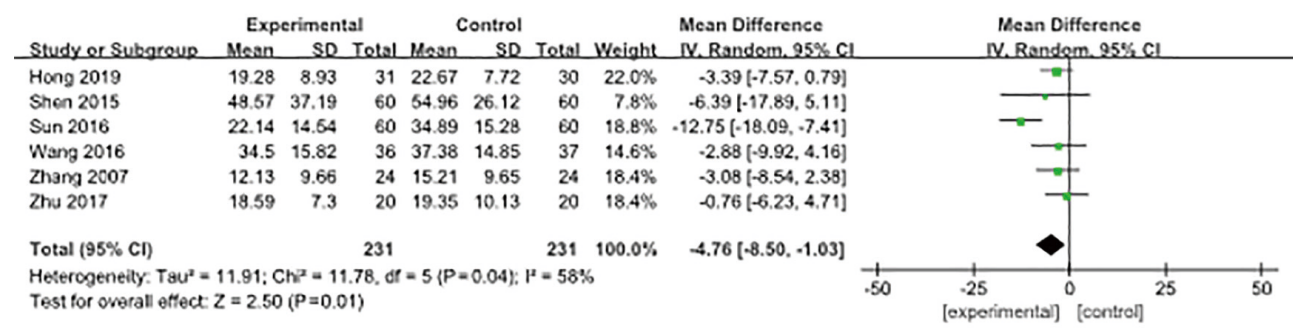

Figure 6 WOMAC score forest plot after acupotomy treatment of KOA. WOMAC, Western Ontario and McMaster Universities Osteoarthritis Index; KOA, knee osteoarthritis; CI, confidence interval; SD, standard deviation.

\section{VAS score after acupotomy treatment of KOA}

In this study, a total of 6 references analyzed the postoperative VAS scores of participants, with a total of $472 \mathrm{KOA}$ patients included, including 236 cases in the experimental group and 236 cases in the control group. The heterogeneity test results $\left(\mathrm{I}^{2}=99 \%, \mathrm{P}<0.00001\right)$ indicated that there was a certain degree of heterogeneity among the studies. Hence, the REM was used for analysis, and the analysis results are shown in Figure 5. The combined effect size was $(M D=-1.44 ; 95 \%$ CI: -2.47 to $-0.42 ; Z=2.76$; $\mathrm{P}=0.006$ ), and the diamond in the forest diagram was on the left side of the vertical line, which indicated that the postoperative VAS score of the acupotomy treatment was lower than that of the control group, that is, the patients experienced less postoperative pain.

\section{WOMAC score after acupotomy treatment of KOA}

A total of 6 references analyzed the WOMAC scores of participants in this study, with a total of $462 \mathrm{KOA}$ patients included, including 231 cases in the experimental group and 231 cases in the control group. The heterogeneity test results $\left(\mathrm{I}^{2}=58 \%, \mathrm{P}=0.04\right)$ suggested that there was a certain degree of heterogeneity among the studies. Hence, the REM was used for analysis, and the analysis results are shown in Figure 6. The combined effect size of meta-analysis was (MD $=-4.76$; $95 \%$ CI: -8.50 to -1.03 ; $\mathrm{Z}=2.50 ; \mathrm{P}=0.01$ ), and the diamond in the forest diagram was located on the left side of the vertical line, which indicated that the postoperative WOMAC score of the acupotomy treatment was lower than that of the control group.

\section{IL-1 $\beta$ levels after acupotomy treatment of KOA}

In this study, 3 references evaluated postoperative IL$1 \beta$ levels in participants, with a total of 219 KOA patients included, including 110 in the experimental group and 109 in the control group. The heterogeneity test results $\left(\mathrm{I}^{2}=74 \%, \mathrm{P}=0.02\right)$ suggested that there was a certain degree of heterogeneity among the studies. Hence, the REM was used for analysis, and the analysis results are shown in Figure 7. The combined effect size of meta-analysis was (MD $=-5.80 ; 95 \%$ CI: -7.16 to $-4.44 ; \mathrm{Z}=8.38 ; \mathrm{P}<0.00001)$, and the diamond in the forest diagram was located on the left side of the vertical line, which indicated the level of IL- $1 \beta$ after acupotomy treatment was lower.

\section{Analysis of the publication bias of acupotomy for KOA}

The software Review Manager 5.3 was used to analyze 


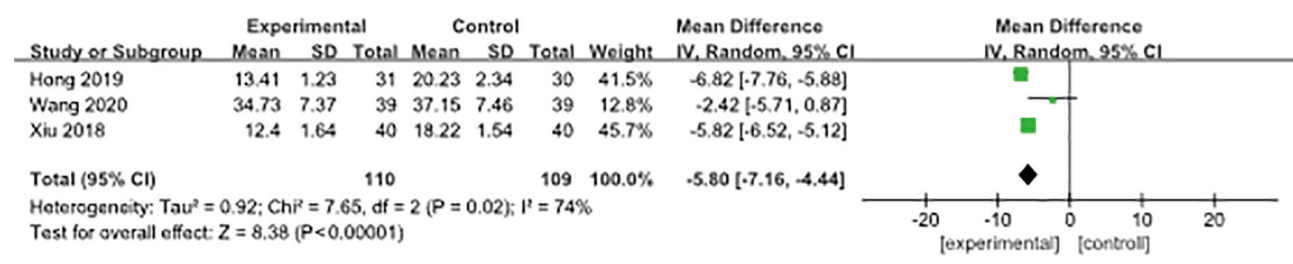

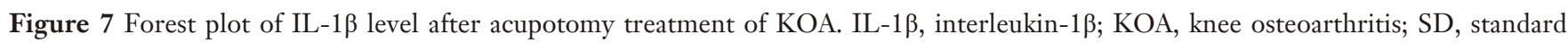
deviation; CI, confidence interval.
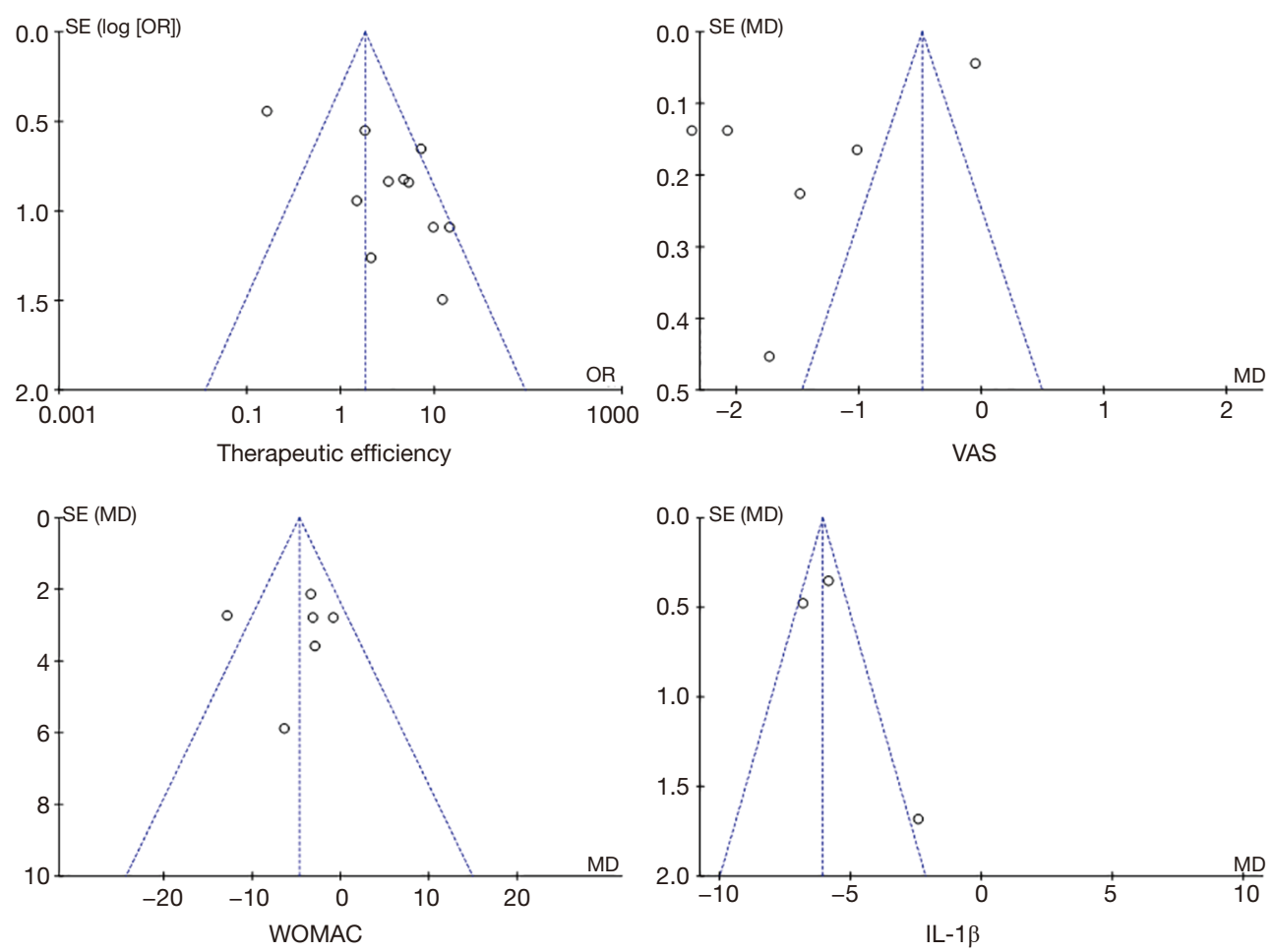

Figure 8 Funnel chart of various outcome evaluation indexes for KOA treated by acupotomy. KOA, knee osteoarthritis; VAS, visual analogue scale; WOMAC, Western Ontario and McMaster Universities Osteoarthritis Index; IL-1, interleukin-1.

the outcome indexes of acupotomy treatment KOA, with a funnel chart drawn, as shown in Figure 8. In the funnel chart of clinical treatment efficiency and WOMAC score, the scattered points representing the literature were basically distributed within the credible interval, and the scattered points were relatively concentrated, which suggested that the publication bias of the literature was low. However, some of the scattered points in the funnel chart of participants' VAS scores and IL- $1 \beta$ levels fell outside the credible interval, and the points were scattered, which suggested that there was a certain publication bias in the literature.

\section{Meta-analysis of acupotomy combined with acupuncture and moxibustion in the treatment of $\mathrm{KO} A$}

\section{Basic information of included literature}

A preliminary search of 5 English databases resulted in a total of 811 related references, of which 238 were retrieved from PubMed, 189 from Embase, 145 from Ovid, 121 from Medline, and 118 from Springer. After the preliminary search, the titles of the references were input into Endnote X6, and 623 references were obtained after duplicates had been eliminated. We then invited 2 researchers to read the title and abstract section of the literature, and 260 references remained according to the inclusion and 


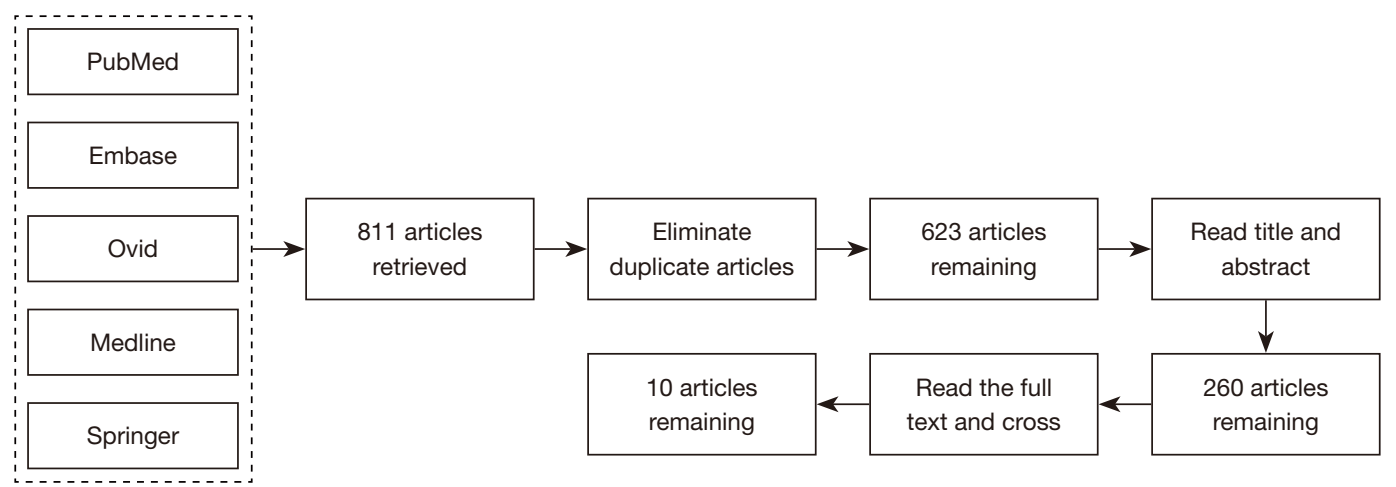

Figure 9 Literature retrieval process of acupotomy combined with acupuncture and moxibustion treatment of KOA. KOA, knee osteoarthritis.

exclusion criteria. Finally, 250 references were further eliminated after their full texts were carefully read, with a total of 10 references (18-27) included in this study. The publication time was from 2011 to 2020, with 983 research cases involved, including 500 in the experimental group and 483 in the control group. The baseline data such as age and disease course of the 2 groups were comparable (Figure 9, Table 2).

\section{The bias risk assessment of included literature}

The Cochrane bias risk assessment tool was used to evaluate the bias risk of the 10 references included in this study, and the software RevMan 5.3 was used to draw the bias risk chart. The results are shown in Figures 10 and 11 . (I) Whether a random sequence was used: all of the 10 included references adopted the random grouping method. Among them, Cheng et al. pointed put the specific random method they used, suggesting low risk, and Wang et al. did not mention specifically which random method was used, suggesting unclear risk. (II) Whether allocation concealment was used: 10 references did not mention whether allocation concealment was applied to participants, suggesting unclear risk. (III) Whether the blind method was used for the subjects: Cheng et al. mentioned in their study that patients had signed an informed consent form, suggesting high risk, and Wang et al. did not mention whether the subjects were blinded, suggesting unclear risk. (IV) Whether the blind method was used for the outcome assessor: 10 references did not mention whether the outcome assessor was blinded, suggesting unclear risk. (V) Whether the research data was complete: the outcome data of 10 references was complete, indicating low risk. (VI) Whether it was a selective report: all of 10 references were not selective reports, indicating low risk. (VII) Whether there were other biases: in the study of Wang et al., there were different numbers of subjects in experimental group and control group, suggesting high risk, and it was undetermined whether there were other biases in the study of Cheng et al., suggesting unclear risk.

\section{The clinical treatment efficiency of acupotomy combined with acupuncture and moxibustion treatment of KOA}

In this study, 9 references analyzed the effective rate of clinical treatment of patients, with a total of 921 KOA patients included, of which 470 cases were in the experimental group, with 451 effective cases, and 451 cases were in the control group, with 366 effective cases. The heterogeneity test results $\left(I^{2}=0 \%, P=0.95\right)$ suggested that the heterogeneity between the studies was small. Hence, the fixed-effects model was used for analysis, and the analysis results are shown in Figure 12. The combined effect size of meta-analysis was ( $\mathrm{MD}=5.72 ; 95 \% \mathrm{CI}$ : 3.39 to 9.64$]$; $\mathrm{Z}=6.54 ; \mathrm{P}<0.00001$ ), and the diamond in the forest diagram was located on the right side of the vertical line, which suggested that the effective rate of acupotomy combined with acupuncture and moxibustion for KOA patients was higher than that of the control group.

\section{The postoperative VAS score of acupotomy combined} with acupuncture and moxibustion treatment for KOA In this study, 6 references analyzed the postoperative VAS scores of participants, with a total of $713 \mathrm{KOA}$ patients included, including 358 cases in the experimental group and 355 cases in the control group. The heterogeneity test results $\left(\mathrm{I}^{2}=99 \%, \mathrm{P}<0.00001\right)$ indicated that there 
Table 2 Basic characteristics of the included literature

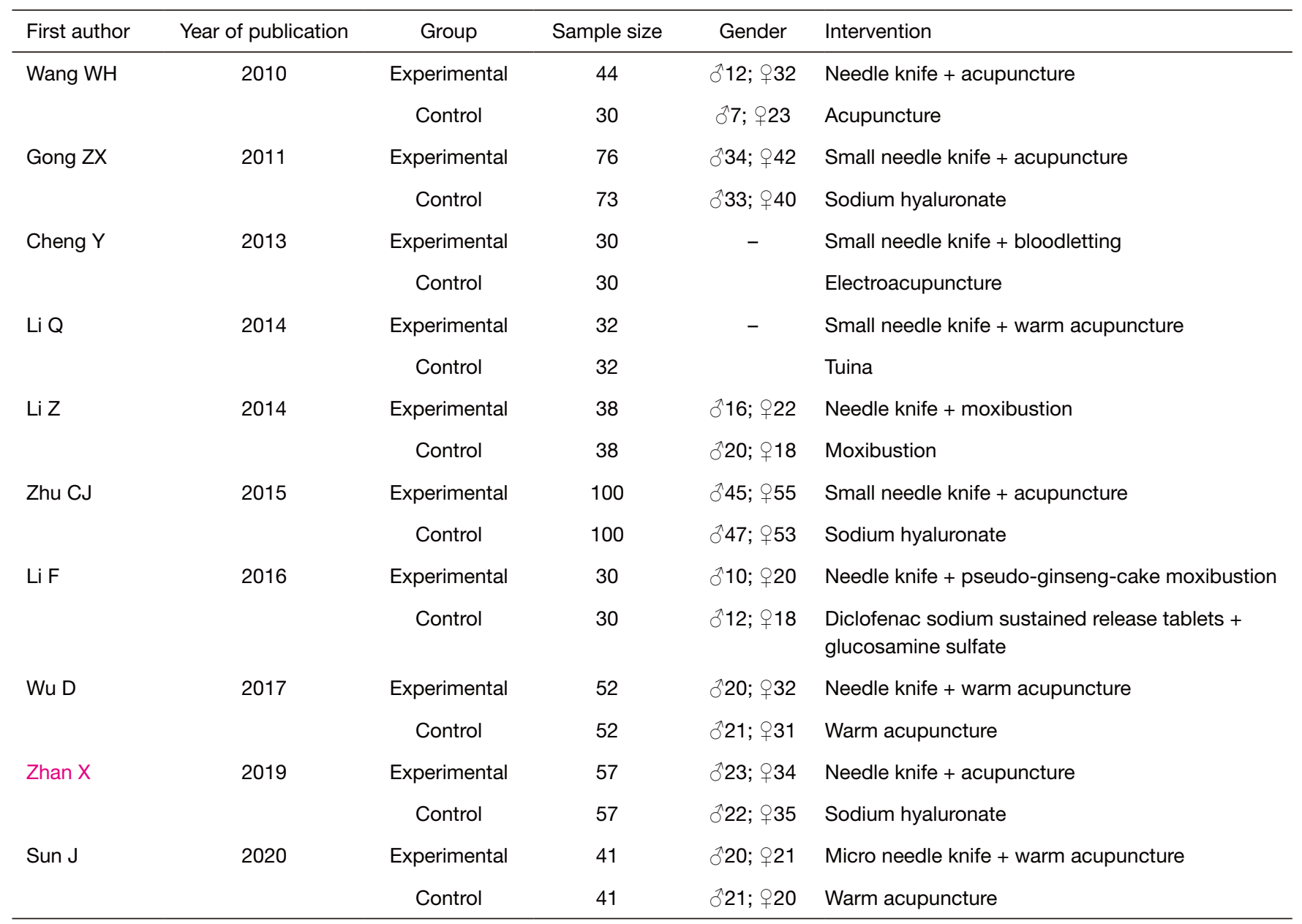

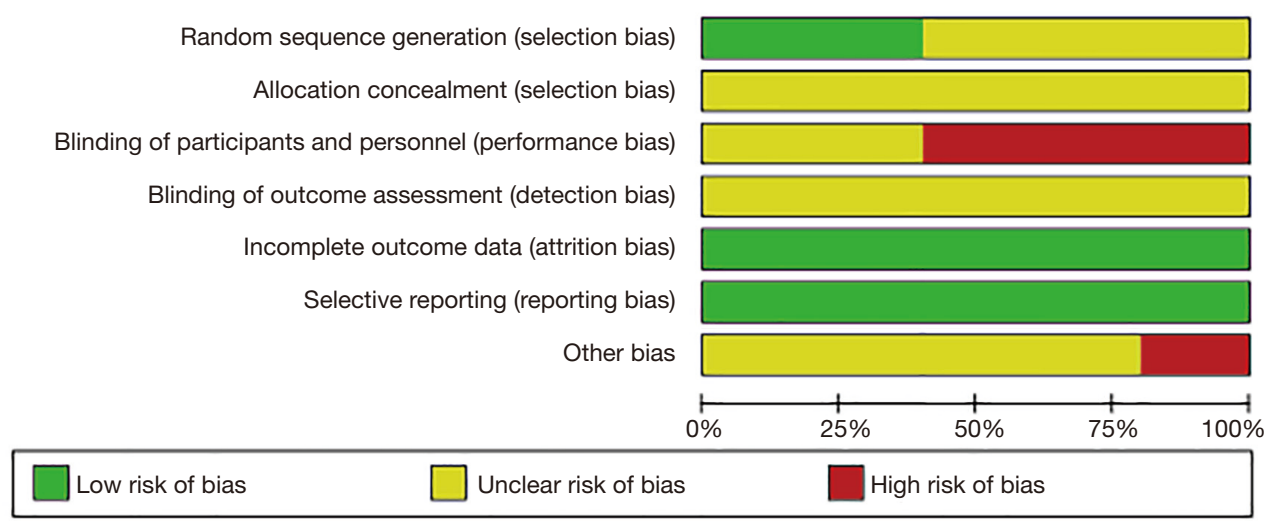

Figure 10 The meta-analysis bias risk assessment bar chart of acupotomy combined with acupuncture and moxibustion for KOA. KOA, knee osteoarthritis. 


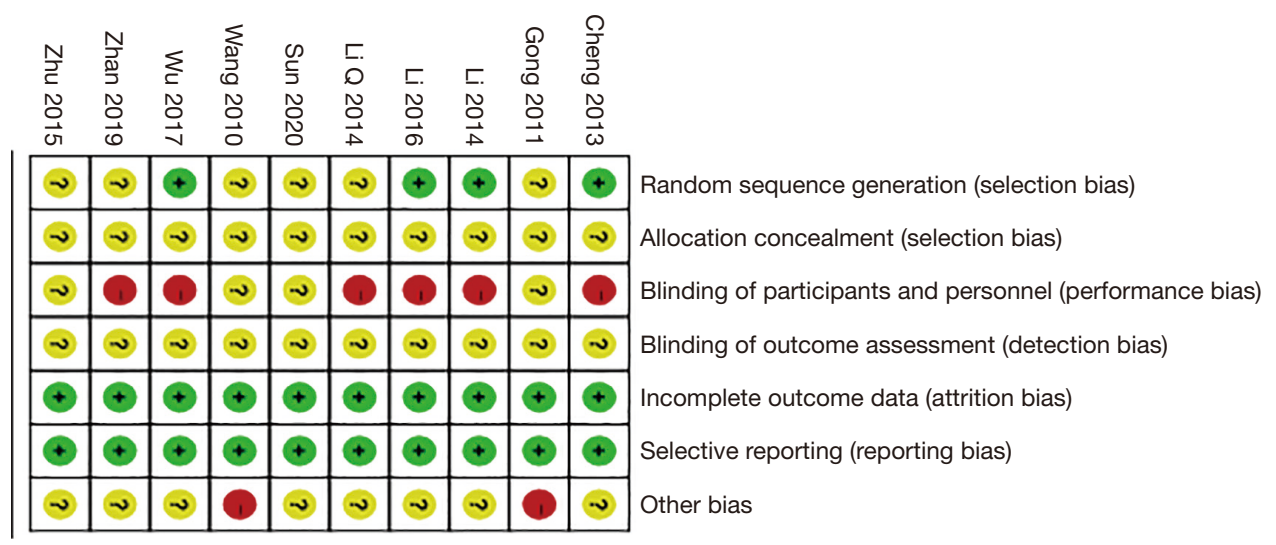

Figure 11 Meta-analysis risk bias assessment graph in the treatment of KOA by acupotomy combined with acupuncture and moxibustion. KOA, knee osteoarthritis.

\begin{tabular}{|c|c|c|c|c|c|c|c|c|c|c|}
\hline Study or Subareue & \multicolumn{2}{|c|}{ Experimental } & \multicolumn{2}{|c|}{ Control } & Weight & $\begin{array}{l}\text { Odds Ratio } \\
M-H . F i x e d .25 \% \mathrm{Cl}\end{array}$ & & \multicolumn{2}{|c|}{$\begin{array}{c}\text { Odds Ratio } \\
M-H . F i x e d .25 \% \mathrm{Cl}\end{array}$} & \\
\hline Cheng 2013 & 29 & 30 & 28 & 30 & $6.4 \%$ & $2.07[0.18,24.15]$ & & & & \\
\hline Gong 2011 & 75 & 76 & 63 & 73 & $5.8 \%$ & $11.90[1.48,95.55]$ & & & & \\
\hline Li 2016 & 37 & 38 & 28 & 36 & $5.2 \%$ & $10.57[1.25,89.50]$ & & & & \\
\hline LIQ 2014 & 30 & 32 & 25 & 32 & $10.8 \%$ & $4.20[0.80 .22 .06]$ & & & & \\
\hline Sun 2020 & 37 & 41 & 30 & 41 & $20.2 \%$ & $3.39[0.98,11.74]$ & & & & \\
\hline Wang 2010 & 41 & 44 & 23 & 30 & $12.9 \%$ & $4.16[0.98,17.66]$ & & & & \\
\hline Wu 2017 & 51 & 52 & 45 & 52 & $6.0 \%$ & $7.93[0.94,66.98]$ & & & & \\
\hline Zhan 2019 & 55 & 57 & 45 & 57 & $10.9 \%$ & $7.33[1.56 .34 .48]$ & & & & \\
\hline Zhu 2015 & 96 & 100 & 79 & 100 & $21.8 \%$ & $6.38[2.10,19.36]$ & & & & \\
\hline Total $(95 \% \mathrm{Cl})$ & & 470 & & 451 & $100.0 \%$ & $5.72[3.39,9.64]$ & & & & \\
\hline Total events & 451 & & 366 & & & & & & & \\
\hline $\begin{array}{l}\text { Heterogeneity: } \mathrm{Chi}^{2}= \\
\text { Tost for overall offoct }\end{array}$ & $\begin{array}{l}68 . \mathrm{df}=8 \\
z=6.54(F\end{array}$ & $\begin{array}{l}(P=0.9 \\
<0.000\end{array}$ & $\begin{array}{l}95) ; 1^{2}=0 \\
01)\end{array}$ & & & & 0.001 & $\begin{array}{l}0.1 \\
\text { crimental] }\end{array}$ & $\begin{array}{lr}1 & 10 \\
\text { [control] }\end{array}$ & 1000 \\
\hline
\end{tabular}

Figure 12 Forest plot of clinical treatment effective rate of acupotomy combined with acupuncture and moxibustion for KOA. KOA, knee osteoarthritis; CI, confidence interval.

\begin{tabular}{|c|c|c|c|c|c|c|c|c|c|c|c|}
\hline \multirow[b]{2}{*}{ Study or Subgroup } & \multicolumn{3}{|c|}{ Experimental } & \multicolumn{2}{|c|}{ Control } & \multirow{2}{*}{\multicolumn{2}{|c|}{ Iotal Weight }} & \multirow{2}{*}{$\begin{array}{l}\text { Mean Difference } \\
\text { IV. Bandem. } 25 \% \mathrm{cl}\end{array}$} & \multirow{2}{*}{\multicolumn{3}{|c|}{$\begin{array}{c}\text { Mean Difference } \\
\text { IV. Randem, } 25 \% \mathrm{Cl}\end{array}$}} \\
\hline & Usan & SD & Iotal & Mean & So & & & & & & \\
\hline Gong 2011 & 2.11 & 0.57 & 76 & 4.67 & 0.45 & 73 & $16.9 \%$ & $-2.56[-2.72,-2.40]$ & - & & \\
\hline LiQ 2014 & 1.25 & 0.88 & 32 & 2.33 & 1.12 & 32 & $15.7 \%$ & $-1.08[-1.57,-0.59]$ & $=$ & & \\
\hline Sun 2020 & 2.82 & 0.36 & 41 & 3.47 & 0.74 & 41 & $16.7 \%$ & $-0.65[-0.90,-0.40]$ & - & & \\
\hline Wu 2017 & 2.8 & 0.3 & 52 & 3.7 & 0.5 & 52 & $16.9 \%$ & $-0.90[-1.06,-0.74]$ & - & & \\
\hline Zhan 2019 & 2.08 & 0.26 & 57 & 4.8 & 0.28 & 57 & $17.0 \%$ & $-2.72[-2.82,-2.62]$ & $=$ & & \\
\hline Zhu 2015 & 2.17 & 0.56 & 100 & 4.51 & 0.32 & 100 & $16.9 \%$ & $-2.34[-2.47,-2.21]$ & - & & \\
\hline Total $(95 \% \mathrm{Cl})$ & & & 358 & & & 355 & $100.0 \%$ & $-1.72[-2.41,-1.03]$ & & & \\
\hline $\begin{array}{l}\text { Heterogeneity: Tau² } \\
\text { Test for overall effect }\end{array}$ & $\begin{array}{l}0.73 ; \mathrm{Ch} \\
Z=4.86\end{array}$ & $\begin{array}{l}F^{2}=55 \\
(P<0 . C\end{array}$ & $\begin{array}{l}1.87,0 \\
0001)\end{array}$ & $=51$ & 0.0 & 01): $1^{2}$ & $2=99 \%$ & & $\begin{array}{c}-5 \\
\text { [experimenta] }\end{array}$ & ${ }^{1}{ }_{\text {[control] }}^{1} 5$ & 10 \\
\hline
\end{tabular}

Figure 13 The postoperative VAS score forest plot of acupotomy combined with acupuncture and moxibustion treatment for KOA. VAS, visual analogue scale; KOA, knee osteoarthritis; CI, confidence interval; SD, standard deviation.

was a certain degree of heterogeneity among the studies. Hence, the REM was used for analysis, and the analysis results are shown in Figure 13. The combined effect size of meta-analysis was (MD $=-1.72 ; 95 \% \mathrm{CI}:-2.41$ to -1.03 ; $Z=4.86 ; P<0.00001)$, and the diamond in the forest plot was on the left of the vertical line, which indicated that the postoperative VAS score of KOA patients treated with acupotomy combined with acupuncture and moxibustion was lower than that of the control group, that is, the patients experienced less postoperative pain. 

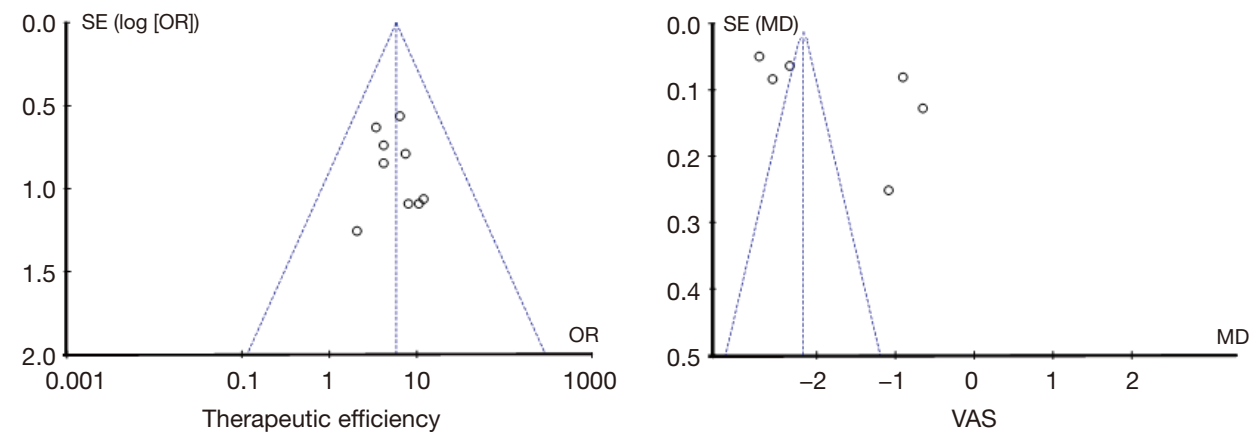

Figure 14 Funnel chart of various outcome evaluation indexes of acupotomy combined with acupuncture and moxibustion treatment for KOA. KOA, knee osteoarthritis; VAS, visual analogue scale.

The publication bias analysis on acupotomy combined with acupuncture and moxibustion treatment for $\mathrm{KOA}$ The software RevMan 5.3 was used to analyze the publication bias of the outcome indexes of the acupotomy combined with acupuncture and moxibustion treatment of KOA, with a funnel chart drawn, as shown in Figure 14. The scatter points representing the literature were all distributed within the credible interval in the funnel chart of the effective clinical treatment, and the scatter points were concentrated, which suggested that the publication bias of the literature was low. However, most of the scattered points fell outside the credible interval in the funnel chart of the participants' VAS scores, and the distribution was relatively scattered, which suggested that the literature had a certain degree of publication bias.

\section{Discussion}

A common type of chronic osteoarthritis, KOA is clinically manifested as knee pain, swelling, and movement disorders. It mostly occurs in the elderly with high incidence. It is held in TCM that the clinical manifestations of KOA belong to the categories of "arthralgia", "bone malaise", and "back and leg pain". It is believed that due to wind-chill dampheat, the meridian is blocked, leading to poor circulation of qi and blood and dystrophy of the joint (28). Clinical TCM treatment methods focus on warming and activating the meridians and promoting blood circulation to dissipate blood stasis. Acupotomy for KOA can not only dredge the meridians, but also separate adhesion tissue, restoring the mechanical balance of knee joints (29). Acupuncture and moxibustion can promote the flow of Qi, blood, and body fluid around the knee joint, and relieve the pain of the patient. The combined treatment of acupotomy and acupuncture and moxibustion can restore the stability of the knee joint and reduce the probability of inflammation, which is of great significance to the treatment of KOA (30).

To realize more accurate systematic evaluation of the therapeutic effects of acupotomy combined with acupuncture and moxibustion on KOA, we conducted a meta-analysis on the therapeutic effects of acupotomy on KOA first, with a total of 13 references included and 1,073 research cases involved. The meta-analysis showed KOA patients treated by acupotomy demonstrated higher clinical treatment efficiency, less postoperative pain, and a reduced IL-1 $\beta$ level. The meta-analysis of the combined treatment for KOA included a total of 10 references, with 983 cases involved. The results showed that KOA patients of the combined treatment exhibited higher clinical treatment efficiency than that of the control group, and lower postoperative VAS scores, suggesting that the combined treatment of KOA can effectively relieve postoperative pain.

\section{Conclusions}

In the study, a systematic analysis was performed to evaluate the clinical therapeutic effects of acupotomy combined with acupuncture and moxibustion in the treatment of KOA. The outcomes showed that acupotomy combined with acupuncture and moxibustion for KOA can increase treatment efficiency and relieve postoperative pain. However, some limitations of the study should be noted. The number of the included references was small. Only 2 indexes were used for comparison, namely, clinical treatment efficiency and VAS score, and only the therapeutic effects at the end of treatment were analyzed, which is not enough to evaluate the long-term therapeutic effects of acupotomy combined with acupuncture and moxibustion. In the follow- 
up, RCTs of an expanded sample size and higher quality are needed to strengthen the findings of this study, so as to accurately evaluate the clinical therapeutic effects of acupotomy combined with acupuncture and moxibustion in the treatment of KOA.

\section{Acknowledgments}

Funding: This study was funded by grants from the Excellent Youth Project of Natural Science Foundation of Heilongjiang Province (YQ2020H028), Excellent Youth Project of Natural Science Foundation of Heilongjiang Province (YQ2020H027), and the Heilongjiang Province Chinese Medicine Research Project (ZHY2020-100).

\section{Footnote}

Reporting Checklist: The authors have completed the PRISMA reporting checklist. Available at https://dx.doi. org/10.21037/apm-21-1083

Conflicts of Interest: All authors have completed the ICMJE uniform disclosure form (available at https://dx.doi. org/10.21037/apm-21-1083). The authors have no conflicts of interest to declare.

Ethical Statement: The authors are accountable for all aspects of the work in ensuring that questions related to the accuracy or integrity of any part of the work are appropriately investigated and resolved.

Open Access Statement: This is an Open Access article distributed in accordance with the Creative Commons Attribution-NonCommercial-NoDerivs 4.0 International License (CC BY-NC-ND 4.0), which permits the noncommercial replication and distribution of the article with the strict proviso that no changes or edits are made and the original work is properly cited (including links to both the formal publication through the relevant DOI and the license). See: https://creativecommons.org/licenses/by-nc-nd/4.0/.

\section{References}

1. Hussain SM, Neilly DW, Baliga S, et al. Knee osteoarthritis: a review of management options. Scott Med J 2016;61:7-16.

2. Wang L, Chen QQ, Tong PJ, et al. Progress on the early diagnosis of knee osteoarthritis. Zhongguo Gu Shang
2016;29:288-291.

3. Kan HS, Chan PK, Chiu KY, et al. Non-surgical treatment of knee osteoarthritis. Hong Kong Med J 2019;25:127-33.

4. Yang M, Jiang L, Wang Q, et al. Traditional Chinese medicine for knee osteoarthritis: An overview of systematic review. PLoS One 2017;12:e0189884.

5. Zhang Y, Guo CQ, Zhang XF, et al. Study of the clinical efficacy of acupotomy for treating Knee Osteoarthritis: a randomized, controlled trial. China Science and Technology Information 2007;(18):219-220,222.

6. Li Z, Chen S. Clinical Observation of Acupotomy in the Treatment of Knee Osteoarthritis. Zhongyi Xuebao 2011;26:19-21.

7. Chen M, Shi XY, Gu YH, et al. Clinical Study on Acupotomy in Treating 60 Cases of Knee Osteoarthritis. Nanjing Zhongyiyao Daxue Xuebao 2011;27:384-6.

8. Shen W, Niu ZJ, Jin Q, et al. Study ni evaluatini nf the efficacy nf acunntnmy therany ii relieviig iitractable naii caused by kiee nstenarthritis. Journal of Clinical and Experimental Medicine 2015;(23):1963-6.

9. Sun K, Bao XM, Song YC, et al. Clinical Efficacy Evaluation on the Treatment of Knee Osteoarthritis by Acupotomy. Journal of Clinical Acupuncture and Moxibustion 2016;32:44-7.

10. Wang XB, Li ZJ. To observe the curative effect of the treatment of knee osteoarthritis with Acupotomy. Journal of Clinical Medical Literature (Electronic Edition) 2016;3:11699-700.

11. Zhong ZP, Sun YX. Clinical efficacy of acupotomy in relieving intractable pain of knee osteoarthritis. Journal of Clinical Medical Literature (Electronic Edition) 2017;4:9113-4.

12. Wang XC. Small Needle-knife in Treating 50 Cases of Knee Osteoarthritis. Western Journal of Traditional Chinese Medicine 2017;30:139-41.

13. Zhu DY, Wu MX. Clinical Observation and Mechanism of Acupotomy in the Treatment of Knee Osteoarthritis. China Health Standard Management 2017;8:86-8.

14. Xiu ZB, Zhang CX, Liu H, et al. Clinical Effect Observation and Mechanism Discussion on Needle Knife Release in Treatment of Knee osteoarthritis. Liaoning Zhongyiyao Daxue Xuebao 2018;20:15-18.

15. Hong HQ, Zhang CR, Chen DC, et al. Clinical effect of needle-knife in the treatment of knee osteoarthritis. Zhongguo Yiyao Daobao 2019;16:162-165,169.

16. Sun XJ. Clinical Application of Acupotomology in the Treatment of Knee Osteoarthritis. World Latest Medicine 
Information 2019;19:19-20.

17. Wang Y, Wang N, Qiao YY, et al. Clinical Study on Needle Knife for Knee Osteoarthritis. Journal of New Chinese Medicine 2020;52:116-9.

18. Wang WH, Li YX. Clinical observation on treating osteoarthritis of the knee by acupuncture. Clinical Journal of Chinese Medicine 2010;(2):13-4.

19. Gong ZX, Tan XY, Lu M. Clinical observation of needlescalpel accompanied with acupuncture on patients with knee osteoarthritis. Hunan Zhongyi Xueyuan Xuebao 2011;31:69-72.

20. Cheng Y, Wu K, Cheng Z, et al. Randomized controlled study on the treatment of knee osteoarthritis with different acupuncture methods at different stages. Zhongguo Zhen Jiu 2013;33:508-12.

21. Li Q, Zhu Y. The Radom Parallel Control Study of Acupuncture And Moxibustion, Acupotomology Therapy Combined With Massage In The Treatment Of Knee Osteoarthritis. Journal of Practical Traditional Chinese Internal Medicine 2014;28:120-3.

22. Li Z. Needle-knife Therapy Combined with Moxibustion in Treating Yang Deficiency Type Knee Osteoarthritis. Zhongyi Xuebao 2014;(7):1067-8.

23. Zhu CJ. Clinical study on acupuncture treatment of acupotomy combined with knee osteoarthritis. Yiayao Qianyan 2015;5:44-5.

24. Li F, Jiang TX, Yang J. Needle-knife combined with pseudo-ginseng-cake moxibustion for primary knee

Cite this article as: $\mathrm{Qu} \mathrm{B}, \mathrm{Wu} \mathrm{X}$, Liu H, Cai W, Wang G, Song H, Wang F. Meta-analysis and systematic review of acupotomy combined with puncture and moxibustion in the treatment of knee osteoarthritis. Ann Palliat Med 2021;10(6):6637-6649. doi: 10.21037/apm-21-1083 osseous arthritis of blood stasis syndrome:a randomized controlled trial. Zhongguo Zhen Jiu 2016;36:799-802.

25. Wu D. Curative Effect Observation of Warm Needle Acupuncture Combined with Needle Knife Therapy on Gonarthritis. Clin Res 2017;25:160-1.

26. Zhan XJ, Huang MJ, Cao Y, et al. Clinical observation on treating knee degenerative osteoarthritis by acupotomy plus acupuncture. Clinical Journal of Chinese Medicine 2019;11:95-8.

27. Sun J, Li F, Xue ZH, et al. Effects of Warm Acupuncture Combined with Micro-Needle Knife on Clinical Symptoms and Range of Motion in Patients with Knee Osteoarthritis (Wind-Cold and Dampness Type). Zhonghua Zhongyiyao Xuekan 2020;38:217-20.

28. Gu YG, Jiang H. Correlation between synovitis and traditional Chinese medicine syndromes of knee osteoarthritis in WORMS score. Zhongguo Gu Shang 2019;32:1108-11.

29. Jin XF, Guo CQ, Liu YF. Brief Talking about Mechanism of Mechanics' Signal Transduction of Knee Osteoarthritis Treated by Acupotome. Zhongguo Zhongyi Jichu Yixue Zazhi 2011;17:1363-1364,1370.

30. Zhao PQ, Guan XF. Research Progress of Acupuncture and Moxibustion in the Treatment of Knee Osteoarthritis. Journal of Clinical Acupuncture and Moxibustion 2015;(3):79-82.

(English Language Editor: J. Jones) 